\title{
Quality and Financial Outcomes From Gainsharing for Inpatient Admissions: A Three-Year Experience
}

\author{
I. Michael Leitman, mD \\ Ruth Levin, MPA \\ Michael J. Lipp, mD \\ Latha Sivaprasad, mD \\ Christine J. \\ Karalakulasingam, mD \\ David S. Bernard, MD \\ Patricia Friedmann, ms \\ David J. Shulkin, MD
}

Beth Israel Medical Center, Albert Einstein College of Medicine, New York, New York.

Disclosure: None of the authors have any financial relationship with the commercial products described herein.

BACKGROUND: Gainsharing is a way to provide incentives to physicians to decrease hospital costs without compromising quality.

METHODS: A pay-for-performance program was instituted over a three-year period from July 2006 to June 2009. Baseline length of stay (LOS) and case costs were developed during the year prior to the inception of the program. Best practice norms (BPNs) were established at the top 25th percentile of physicians for each all patient refined (APR)-diagnosis related group (DRG). Hospital costs were analyzed in several areas, including operating room charge (OR), supplies and implants, nursing and per-diem room costs. Payments were based upon case level performance compared to BPN's and the physician's historic performance. Eligible cases included commercial insurance only for the first 2 years but Medicare cases were included after October 2008 resulting from a Centers for Medicare and Medicaid Services (CMS)-approved demonstration project. Payments to physicians required meeting quality thresholds, including chart completion, and compliance with core measures.

RESULTS: A total of 184 (54\%) physicians enrolled into the program. There was a $\$ 25.1$ million reduction in hospital costs during the 3 years ( $\$ 16$ million from participating and $\$ 9.1$ million from non-participating physicians, $P<0.01$ ). Most cost reductions were attributed to reduced LOS and reductions in medical supply costs. Total physician payouts were over $\$ 2$ million (average $\$ 1,866$ per quarter). Delinquent medical records decreased from an average of $43 \%$ in the second quarter 2006 to $30 \%(P<0.0001)$ in the second quarter 2009. Quality measures improved during the study period but not by a statistical significance.

CONCLUSIONS: Gainsharing provided an incentive for physicians to reduce hospital costs while maintaining hospital quality. Journal of Hospital Medicine 2010;5:501-507. @ 2010 Society of Hospital Medicine.

KEYWORDS: core measures, financial outcome, gainsharing, healthcare delivery systems, hospital costs, pay-for-performance, physician incentives, quality.

Hospitals are challenged to improve quality while reducing costs, yet traditional methods of cost containment have had limited success in aligning the goals of hospitals and physicians. Physicians directly control more than $80 \%$ of total medical costs. ${ }^{1}$ The current fee-for-service system encourages procedures and the use of hospital resources. Without the proper incentives to gain active participation and collaboration of the medical staff in improving the efficiency of care, the ability to manage medical costs and improve hospital operational and financial performance is hampered. A further challenge is to encourage physicians to improve the quality of care and maintain safe medical practice. While several examples of pay-for-performance ( $\mathrm{P} 4 \mathrm{P})$ have previously been attempted to increase efficiency, gainsharing offers real opportunities to achieve these outcomes.

Previous reports regarding the results of gainsharing programs describe its use in outpatient settings and its limited ability to reduce costs for inpatient care for surgical implants such as coronary stents ${ }^{2}$ or orthopedic prostheses. ${ }^{3}$ The present study represents the largest series to date using a gainsharing model in a comprehensive program of inpatient care at a tertiary care medical center.

\section{Patients and Methods}

Beth Israel Medical Center is a 1000-bed tertiary care university-affiliated teaching hospital, located in New York City. The hospital serves a large and ethnically diverse community predominantly located in the lower east side of Manhattan and discharged about 50,000 patients per year during the study period of July 2006 through June 2009.

Applied Medical Software, Inc. (AMS, Collingswood, NJ) analyzed hospital data for case mix and severity. To establish "best practice norms" (BPNs), AMS used inpatient discharge data (UB-92) to determine costs by APR-DRG's ${ }^{4}$ during calendar year 2005, prior to the inception of the program to establish BPNs. Costs were allocated into specific 


\section{TABLE 1. Hospital Cost Allocation Areas in the} Gainsharing Program

\begin{tabular}{ll} 
Per diem hospital bed cost & Pharmacy \\
Critical care (ICU and CCU) & Laboratory \\
Medical surgical supplies and implants & Cardiopulmonary care \\
Operating room costs & Blood bank \\
Radiology & Intravenous therapy \\
\hline
\end{tabular}

Abbreviations: CCU, coronary care unit; ICU, intensive care unit

areas listed in Table 1. A minimum of 10 cases was necessary in each DRG. Cost outliers (as defined by the mean cost of the APR DRG plus 3 standard deviations) were excluded. These data were used to establish a baseline for each physician and a BPN, which was set at the top 25th percentile for each specific APR DRG. BPNs were determined after exclusions using the following criteria:

1. Each eligible physician had to have at least 10 admissions within their specialty;

2. Each eligible DRG had to have at least 5 qualifying physicians within a medical specialty;

3. Each eligible APR DRG had to have at least 3 qualifying admissions;

4. If the above criteria are met, the BPN was set at the mean of the top 25th percentile of physicians $(25 \%$ of the physicians with the lowest costs).

Once BPNs were determined, patients were grouped by physician and compared to the BPN for a particular APR DRG. All patients of participating physicians with qualifying APR DRGs were included in the analysis reports summarizing these results, computed quarterly and distributed to each physician. Obstetrical and psychiatric admissions were excluded in the program. APR DRG data for each physician was compared from year to year to determine whether an individual physician demonstrated measurable improvement in performance.

The gainsharing program was implemented in 2006. Physician participation was voluntary. Payments were made to physicians without any risk or penalties from participation. Incentives were based on individual performance. Incentives for nonsurgical admissions were intended to offset the loss of physician income related to more efficient medical management and a reduced hospital length of stay (LOS). Income for surgical admissions was intended to reward physicians for efficient preoperative and postoperative care.

The methodology provides financial incentives for physicians for each hospital discharge in 2 ways:

1. Improvement in costs per case against their own historical performance;

2. Cost per case performance compared to BPN.

In the first year of the gainsharing program, two thirds of the total allowable incentive payments were allocated to physicians' improvement, with one third based on a perform- ance metric. Payments for improvement were phased out over the first 3 years of the gainsharing program, with payments focused fully on performance in Year 3. Cases were adjusted for case-mix and severity of illness (four levels of APR DRG). Physicians were not penalized for any cases in which costs greatly exceeded BPN. A floor was placed at the BPN and no additional financial incentives were paid for surpassing it. Baselines and BPNs were recalculated yearly.

A key aspect of the gainsharing program was the establishment of specific quality parameters (Table 2) that need to be met before any incentive payments were made. A committee regularly reviewed the quality performance data of each physician to determine eligibility for payments. Physicians were considered to be ineligible for incentive compensation until the next measurement period if there was evidence of failure to adequately meet these measures. At least $80 \%$ compliance with core measures (minimum 5 discharges in each domain) was expected. Infectious complication rates were to remain not more than 1 standard deviation above National Healthcare Safety Network rates during the same time period. In addition, payments were withheld from physicians if it was found that the standard of care was not met for any morbidity or mortality that was peer reviewed or if there were any significant patient complaints. Readmission rates were expected to remain at or below the baseline established during the previous 12 months by DRG.

Employed and private practice community physicians were both eligible for the gainsharing program. Physician participation in the program was voluntary. All patients admitted to the Medical Center received notification on admission about the program. The aggregate costs by DRG were calculated quarterly. Savings over the previous year-if any-were calculated. A total of $20 \%$ of the savings was used to administer the program and for incentive payments to physicians.

From July 1, 2006 through September 2008, only commercial managed care cases were eligible for this program. As a result of the approval of the gainsharing program as a demonstration project by the Centers for Medicare and Medicaid Services (CMS), Medicare cases were added to the program starting October 1, 2008.

\section{Physician Payment Calculation Methodology \\ Performance Incentive}

The performance incentive was intended to reward demonstrated levels of performance. Accordingly, a physician's share in hospital savings was in proportion to the relationship between their individual performance and the BPN. This computation was the same for both surgical and medical admissions. The following equation illustrates the computation of performance incentives for participating physicians:

$\left\{\begin{array}{c}\text { 90th Percentile BPN Cost-Physician's Actual Cost } \\ \text { 90th Percentile Cost-Best Practice Norm Cost }\end{array}\right\}$ Maximum Performance Incentive 


\section{TABLE 2. Quality Factors Used to Determine Physician Payment in Gainsharing Program}

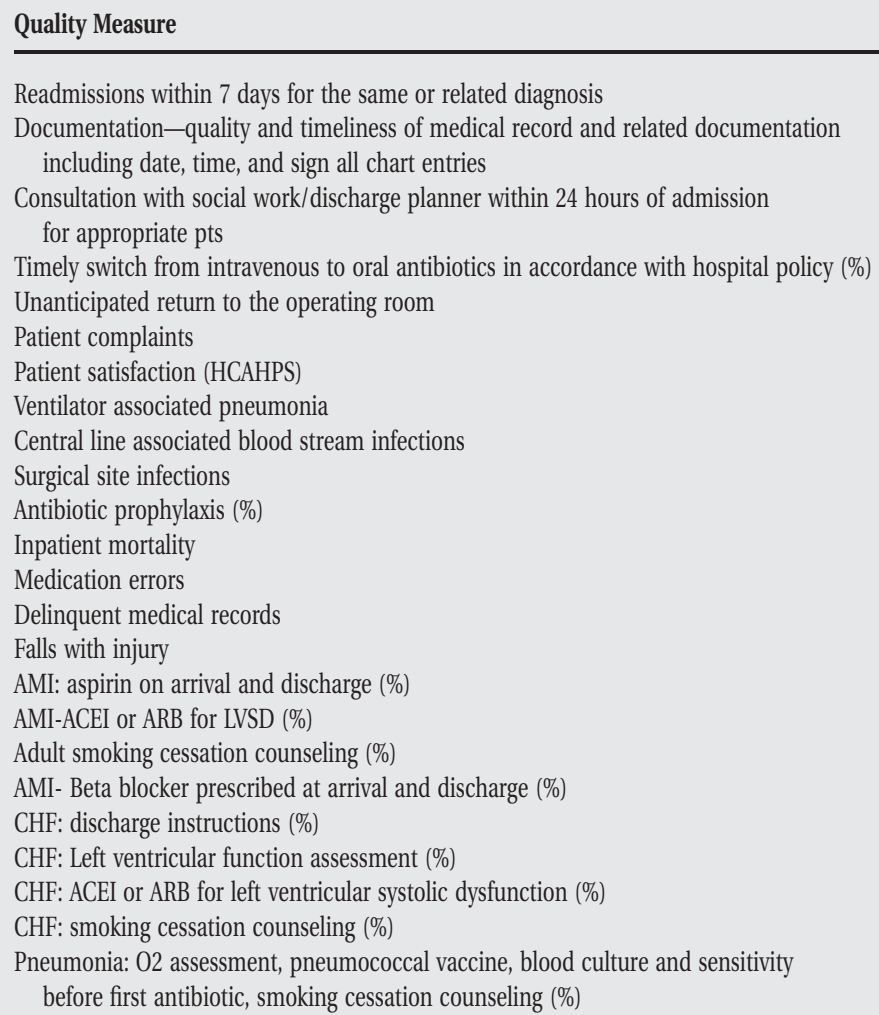

Readmissions within 7 days for the same or related diagnosis

Documentation-quality and timeliness of medical record and related documentation including date, time, and sign all chart entries

Consultation with social work/discharge planner within 24 hours of admission for appropriate pts

Timely switch from intravenous to oral antibiotics in accordance with hospital policy (\%)

Unanticipated return to the operating room

Patient complaints

Patient satisfaction (HCAHPS)

Ventilator associated pneumonia

Central line associated blood stream infections

Surgical site infections

Antibiotic prophylaxis (\%)

Inpatient mortality

Medication errors

Delinquent medical records

Falls with injury

AMI: aspirin on arrival and discharge (\%)

AMI-ACEI or ARB for LVSD (\%)

Adult smoking cessation counseling (\%)

AMI- Beta blocker prescribed at arrival and discharge (\%)

CHF: discharge instructions (\%)

CHF: Left ventricular function assessment (\%)

CHF: ACEI or ARB for left ventricular systolic dysfunction (\%)

CHF: smoking cessation counseling (\%)

Pneumonia: 02 assessment, pneumococcal vaccine, blood culture and sensitivity

before first antibiotic, smoking cessation counseling (\%)

Decrease, or less than $10 \%$ of discharges

No more than $20 \%$ of average monthly discharged medical records incomplete for more than 30 days

$>80 \%$ of all appropriate cases

$>80$
Decr
Decr
$>75 \%$
Decr
Decr
Decr
$>80$
Decr
Decr
$<5$
Decr
$>80$
$>80$
$>80$
$>80$
$>80$
$>80$
$>80$
$>80$
$>80$

Decrease or $<5 \%$

Decrease

$>75 \%$ physician domain

Decrease or $<5 \%$

Decrease or $<5$ per 1000 catheter days.

Decrease or within 1 standard deviation of NHSN

Decrease or $<1 \%$

Decrease or $<1 \%$

$<5$ charts delinquent more than 30 days

Decrease or $<1 \%$

Abbreviations: ACEI, Angiotensin converting enzyme inhibitor; AMI, Acute myocardial infarction; ARB, Angiotensin II receptor blockers; CHF, Congestive heart failure; HCAHPS, Hospital consumer assessment of healthcare providers and systems; LVSD, left ventricular systolic dysfunction; NHSN, Center for Disease Control (CDC) National Healthcare Safety Network.

This computation was made at the specific severity level for each hospital discharge. Payment for the performance incentive was made only to physicians at or below the 90th percentile of physicians.

\section{Improvement Incentive}

The improvement incentive was intended to encourage positive change. No payments were made from the improvement incentive unless an individual physician demonstrated measurable improvement in operational performance for either surgical or medical admissions. However, because physicians who admitted nonsurgical cases experienced reduced income as they help the hospital to improve operational performance, the methodology for calculating the improvement incentive was different for medical as opposed to surgical cases, as shown below.

For Medical DRGs:

For each severity level the following is calculated:

$$
\begin{gathered}
\left\{\begin{array}{c}
\text { Base Year Case Mix Adjusted ALOS-Rate Year } \\
\text { Case Mix Adjusted ALOS }
\end{array}\right\} \\
\text { X } \\
\text { Per Diem } \\
\text { X } \\
\text { Rate year Admissions }
\end{gathered}
$$

For Surgical DRGs:

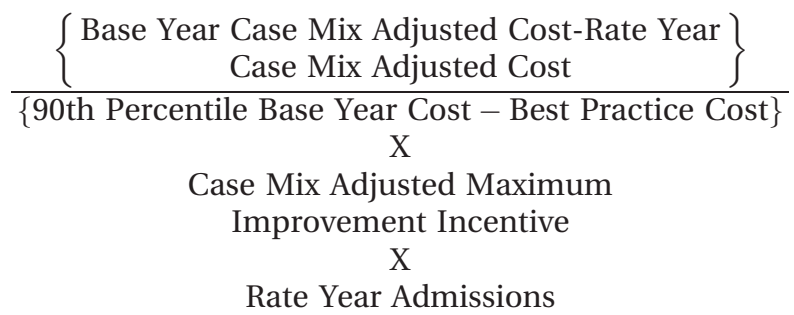

Cost savings were calculated quarterly and defined as the cost per case before the gainsharing program began minus the actual case cost by APR DRG. Student's $t$-test was used for continuous data and the categorical data trends were analyzed using Mantel-Haenszel Chi-Square.

At least every 6 months, all participating physicians received case-specific and cost-centered data about their discharges. They also received a careful explanation of opportunities for financial or quality improvement.

\section{Results}

Over the 3-year period, 184 physicians enrolled, representing $54 \%$ of those eligible. The remainder of physicians either decided not to enroll or were not eligible due to inadequate 
TABLE 3. Distribution of Cases Among Services for Physicians Participating in Gainsharing

\begin{tabular}{lc} 
Admissions by Service & Number (\%) \\
\hline Cardiology & $4512(15.3)$ \\
Orthopedic surgery & $3994(13.5)$ \\
Gastroenterology & $3214(10.9)$ \\
General surgery & $2908(9.8)$ \\
Cardiovascular surgery & $2432(8.2)$ \\
Pulmonary & $2212(7.5)$ \\
Neurology & $2064(7.0)$ \\
Oncology & $1217(4.1)$ \\
Infectious disease & $1171(4.0)$ \\
Endocrinology & $906(3.1)$ \\
Nephrology & $826(2.8)$ \\
Open heart surgery & $656(2.2)$ \\
Interventional cardiology & $624(2.1)$ \\
Gynecological surgery & $450(1.5)$ \\
Urological surgery & $326(1.1)$ \\
ENT surgery & $289(1.0)$ \\
Obstetrics without delivery & $261(0.9)$ \\
Hematology & $253(0.9)$ \\
Orthopedics-nonsurgical & $241(0.8)$ \\
Rehabilitation & $204(0.7)$ \\
Otolaryngology & $183(0.6)$ \\
Rheumatology & $165(0.6)$ \\
General medicine & $162(0.5)$ \\
Neurological surgery & $112(0.4)$ \\
Urology & $101(0.3)$ \\
Dermatology & $52(0.2)$ \\
Grand total & $29535(100.0)$ \\
\hline Abbreviation: ENT, ear, nose, throat. & \\
\hline & \\
\hline
\end{tabular}

number of index DRG cases or excluded diagnoses. Payer mix was $27 \%$ Medicare and $48 \%$ of the discharges were commercial and managed care. The remaining cases were a combination of Medicaid and self-pay. A total of 29,535 commercial and managed care discharges were evaluated from participating physicians (58\%) and 20,360 similar discharges from non-participating physicians. This number of admissions accounted for $29 \%$ of all hospital discharges during this time period. Surgical admissions accounted for $43 \%$ and nonsurgical admissions for $57 \%$. The distribution of patients by service is shown in Table 3. Pulmonary and cardiology diagnoses were the most frequent reasons for medical admissions. General and head and neck surgery were the most frequent surgical admissions. During the time period of the gainsharing program, the medical center saved \$25.1 million for costs attributed to these cases. Participating physicians saved $\$ 6.9$ million more than nonparticipating physicians $(P=0.02$, Figure 1$)$, but all discharges demonstrated cost savings during the study period. Cost savings (Figure 2) resulted from savings in medical/surgical supplies and implants (35\%), daily hospital costs, (28\%), intensive care unit costs $(16 \%)$ and coronary care unit costs (15\%), and operating room costs $(8 \%)$. Reduction in cost from reduced magnetic resonance imaging (MRI) use was

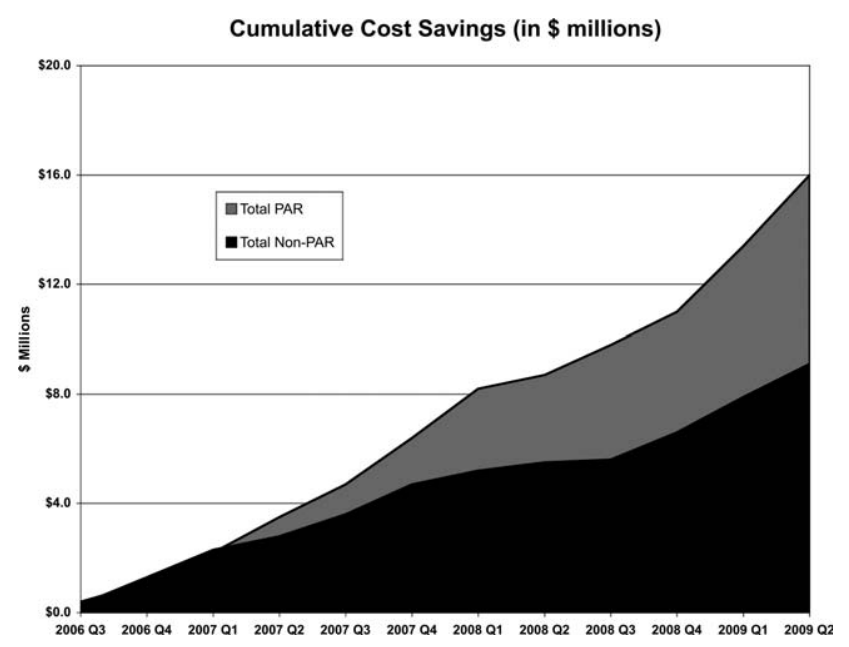

FIGURE 1. Cumulative cost savings (in millions of $\$$ dollars) for participating physicians (PAR) and non-participating physicians (Non-Par) year 2006 to $2009(P=0.02)$.

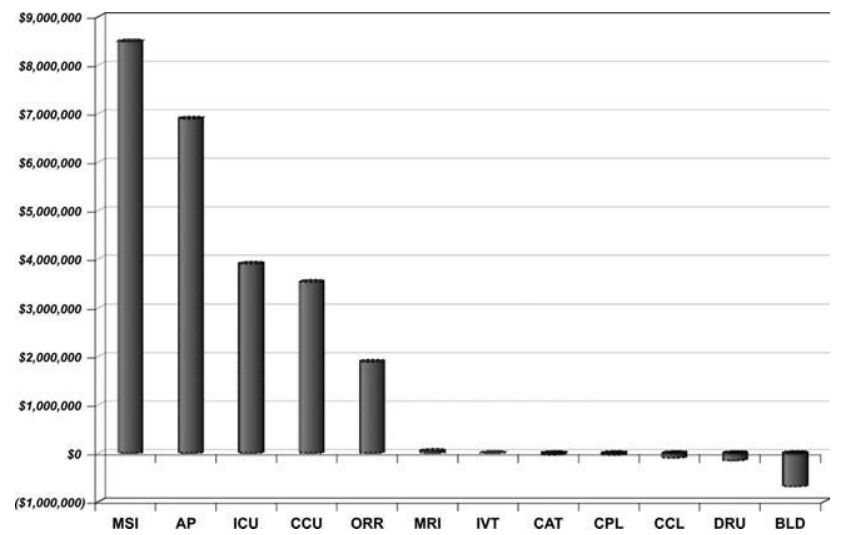

FIGURE 2. Savings ( $\$$ dollars) by cost center. MSI, medical surgical supplies and implants; AP, hospital daily costs; ICU, intensive care unit; CCU, coronary care unit; OR, operating room charges; MRI, magnetic resonance imaging; CT, CT scan; CPL, cardiopulmonary lab; CCL, clinical laboratory; DRU, pharmacy; BLD, blood bank.

not statistically significant. There were minimal increases in costs due to computed tomography (CT) scan use, cardiopulmonary care, laboratory use, pharmacy and blood bank, but none of these reached statistical significance.

Hospital LOS decreased 9.8\% from baseline among participating doctors, while LOS decreased $9.0 \%$ among non-participating physicians; this difference was not statistically significant $(P=0.6)$. Participating physicians reduced costs by an average of $\$ 7,871$ per quarter, compared to a reduction in costs by $\$ 3,018$ for admissions by non-participating physicians $(P<0.0001)$. The average savings per admission for the participating physicians were $\$ 1,835$, and for non-participating physicians were $\$ 1,107$, a difference of $\$ 728$ per admission. Overall, cost savings during the three year period averaged $\$ 105,000$ per physician who participated in the program and $\$ 67,000$ per physician who did not $(P<0.05)$. 
There was not a statistical difference in savings between medical and surgical admissions $(P=0.24)$.

Deviations from quality thresholds were identified during this time period. Some or all of the gainsharing income was withheld from $8 \%$ of participating physicians due to quality issues, incomplete medical records, or administrative reasons. Payouts to participating physicians averaged $\$ 1,866$ quarterly (range $\$ 0-\$ 27,631$ ). Overall, $9.4 \%$ of the hospital savings was directly paid to the participating physicians. Compliance with core measures improved in the following domains from year 2006 to 2009; acute myocardial infarction $94 \%$ to $98 \%$, congestive heart failure $76 \%$ to $93 \%$, pneumonia $88 \%$ to $97 \%$, and surgical care improvement project $90 \%$ to $97 \%$, $(P=0.17)$. There was no measurable increase in 30-day mortality or readmission by APR-DRG. The number of incomplete medical records decreased from an average of $43 \%$ of the total number of records in the second quarter of 2006 to $30 \%$ in the second quarter of $2009(P<0.0001)$. Other quality indicators remained statistically unchanged.

\section{Discussion}

The promise of gainsharing may motivate physicians to decrease hospital costs while maintaining quality medical care, since it aligns physician and hospital incentives. Providing a reward to physicians creates positive reinforcement, which is more effective than warnings against poor performing physicians (carrot vs. stick). ${ }^{5,6}$ This study is the first and largest of its kind to show the results of a gainsharing program for inpatient medical and surgical admissions and demonstrates that significant cost savings may be achieved. This is similar to previous studies that have shown positive outcomes for pay-for-performance programs. ${ }^{7}$

Participating physicians in the present study accumulated almost $\$ 7$ million more in savings than non-participating physicians. Over time this difference has increased, possibly due to a learning curve in educating participating physicians and the way in which information about their performance is given back to them. A significant portion of the hospital's cost savings was through improvements in documentation and completion of medical records. While there was an actual reduction in average length of stay (ALOS), better documentation may also have contributed to adjusting the severity level within each DRG.

Using financial incentives to positively impact on physician behavior is not new. One program in a communitybased hospitalist group reported similar improvements in medical record documentation, as well as improvements in physician meeting attendance and quality goals. ${ }^{8}$ Another study found that such hospital programs noted improved physician engagement and commitment to best practices and to improving the quality of care. ${ }^{9}$

There is significant experience in the outpatient setting using pay-for-performance programs to enhance quality. Millett et al. ${ }^{10}$ demonstrated a reduction in smoking among patients with diabetes in a program in the United Kingdom.
Another study in Rochester, New York that used pay-for-performance incentives demonstrated better diabetes management. $^{11}$ Mandel and Kotagal ${ }^{12}$ demonstrated improved asthma care utilizing a quality incentive program.

The use of financial motivation for physicians, as part of a hospital pay-for-performance program, has been shown to lead to improvements in quality performance scores when compared to non pay-for-performance hospitals. ${ }^{13}$ Berthiaume demonstrated decreased costs and improvements in risk-adjusted complications and risk-adjusted LOS in patents admitted for acute coronary intervention in a pay-forperformance program. ${ }^{14}$ Quality initiatives were integral for the gainsharing program, since measures such as surgical site infections may increase LOS and hospital costs. Core measures related to the care of patients with acute myocardial infarction, heart failure, pneumonia, and surgical prophylaxis steadily improved since the initiation of the gainsharing program. Gainsharing programs also enhance physician compliance with administrative responsibilities such as the completion of medical records.

One unexpected finding of our study was that there was a cost savings per admission even in the patients of physicians who did not participate in the gainsharing program. While the participating physicians showed statistically significant improvements in cost savings, savings were found in both groups. This raises the question as to whether these cost reductions could have been impacted by other factors such as new labor or vendor contracts, better documentation, improved operating room utilization and improved and timely documentation in the medical record. Another possibility is the Hawthorne effect on physicians, who altered their behavior with knowledge that process and outcome measurement were being measured. Physicians who voluntarily sign up for a gainsharing program would be expected to be more committed to the success of this program than physicians who decide to opt out. While this might appear to be a selection bias it does illustrate the point that motivated physicians are more likely to positively change their practice behaviors. However, one might suggest that financial savings directly attributed to the gainsharing program was not the $\$ 25.1$ million saved during the 3 years overall, but the difference between participating and nonparticipating physicians, or $\$ 6.9$ million.

While the motivation to complete medical records was significant (gainsharing dollars were withheld from doctors with more than 5 incomplete charts for more than 30 days) it was not the only reason why the number of delinquent chart percentage decreased during the study period. While the improvement was significant, there are still more opportunities to reduce the number of incomplete charts. Hospital regulatory inspections and periodic physician education were also likely to have reduced the number of incomplete inpatient charts during this time period and may do so in the future. ${ }^{15}$

The program focused on the physician activities that have the greatest impact on hospital costs. While optimizing 
laboratory, blood bank, and pharmacy management decreased hospital costs; we found that improvements in patient LOS, days in an intensive care unit, and management of surgical implants had the greatest impact on costs. Orthopedic surgeons began to use different implants, and all surgeons refrained from opening disposable surgical supplies until needed. Patients in intensive care unit beds stable for transfer were moved to regular medical/surgical rooms earlier. Since the program helped physicians understand the importance of LOS, many physicians increased their rounding on weekends and considered LOS implications before ordering diagnostic procedures that could be performed as an outpatient. Nurses, physician extenders such as physician assistants, and social workers have played an important role in streamlining patient care and hospital discharge; however, they were not directly rewarded under this program.

There are challenges to aligning the incentives of internists compared to procedure-based specialists. This may be that the result of surgeons receiving payment for bundled care and thus the incentives are already aligned. The incentive of the program for internists, who get paid for each per daily visit, was intended to overcome the lost income resulting from an earlier discharge. Moreover, in the present study, only the discharging physician received incentive payments for each case. Patient care is undoubtedly a team effort and many physicians (radiologists, anesthesiologists, pathologists, emergency medicine physicians, consultant specialty physicians, etc.) are clearly left out in the present gainsharing program. Aligning the incentives of these physicians might be necessary. Furthermore, the actions of other members of the medical team and consultants, by their behaviors, could limit the incentive payments for the discharging physician. The discharging physician is often unable to control the transfer of a patient from a high-cost or severity unit, or improve the timeliness of consulting physicians. Previous authors have raised the issue as to whether a physician should be prevented from payment because of the actions of another member of the medical team. $^{16}$

Ensuring a fair and transparent system is important in any pay-for-performance program. The present gainsharing program required sophisticated data analysis, which added to the costs of the program. To implement such a program, data must be clear and understandable, segregated by DRG and severity adjusted. But should the highest reward payments go to those who perform the best or improve the most? In the present study, some physicians were consistently unable to meet quality benchmarks. This may be related to several factors, 1 of which might be a particular physician's case mix. Some authors have raised concerns that pay-for-performance programs may unfairly impact physicians who care for more challenging or patients from disadvantaged socioeconomic circumstances. ${ }^{17}$ Other authors have questioned whether widespread implementation of such a program could potentially increase healthcare disparities in the community. ${ }^{18}$ It has been suggested by Greene and Nash that for a program to be successful, physicians who feel they provide good care yet but are not rewarded should be given an independent review. ${ }^{16}$ Such a process is important to prevent resentment among physicians who are unable to meet benchmarks for payment, despite hard work. ${ }^{19}$ Conversely, other studies have found that many physicians who receive payments in a pay-for-performance system do not necessarily consciously make improvement to enhance financial performance. ${ }^{20}$ Only $54 \%$ of eligible physicians participated in the present gainsharing program. This is likely due to lack of understanding about the program, misperceptions about the ethics of such programs, perceived possible negative patient outcome, conflict of interest and mistrust. ${ }^{21,22}$ This underscores the importance of providing understandable performance results, education, and a physician champion to help facilitate communication and enhanced outcomes. What is clear is that the perception by participating physicians is that this program is worthwhile as the number of participating physicians has steadily increased and it has become an incentive for new providers to choose this medical center over others.

In conclusion, the results of the present study show that physicians can help hospitals reduce inpatients costs while maintaining or improving hospital quality. Improvements in patient LOS, implant costs, overall costs per admission, and medical record completion were noted. Further work is needed to improve physician education and better understand the impact of uneven physician case mix. Further efforts are necessary to allow other members of the health care team to participate and benefit from gainsharing.

\section{Address for correspondence and reprint requests:}

I. Michael Leitman, MD, Beth Israel Medical Center, 10 Union Square East, Suite 2M, New York, NY 10003; Telephone: 212-8448570; Fax: 212-844-8440; E-mail: Mleitman@chpnet.org Received 11 February 2010; revision received 22 March 2010; accepted 4 April 2010 .

\section{References}

1. Leff B, Reider L, Frick KD, et al. Guided care and the cost of complex healthcare: a preliminary report. Am J Manag Care. 2009;15(8):555-559.

2. Ketcham JD, Furukawa MF. Hospital-physician gainsharing in cardiology. Health Aff (Millwood). 2008;27(3):803-812.

3. Dirschl DR, Goodroe J, Thornton DM, Eiland GW. AOA Symposium. Gainsharing in orthopaedics: passing fancy or wave of the future? J Bone Joint Surg Am. 2007;89(9):2075-2083.

4. All Patient Defined Diagnosis Related Groups ${ }^{\mathrm{TM}}-3 \mathrm{M}$ Health Information Systems, St Paul, MN.

5. Leff B, Reider L, Frick KD, et al. Guided care and the cost of complex healthcare: a preliminary report. Am J Manag Care. 2009;15(8):555-559.

6. Doyon C. Best practices in record completion. J Med Pract Manage. 2004; 20(1):18-22.

7. Curtin K, Beckman H, Pankow G, et al. Return on investment in pay for performance: a diabetes case study. J Healthc Manag. 2006;51(6):365-374; discussion 375-376.

8. Collier VU. Use of pay for performance in a community hospital private hospitalists group: a preliminary report. Trans Am Clin Climatol Assoc. 2007;188:263-272. 
9. Williams J. Making the grade with pay for performance: 7 lessons from best-performing hospitals. Healthc Financ Manage. 2006;60(12):79-85.

10. Millett C, Gray J, Saxena S, Netuveli G, Majeed A. Impact of a pay-forperformance incentive on support for smoking cessation and on smoking prevalence among people with diabetes. CMAJ. 2007;176(12):1705-1710.

11. Young GJ, Meterko M, Beckman H, et al, Effects of paying physicians based on their relative performance for quality. J Gen Intern Med. 2007; 22(6):872-876

12. Mandel KE, Kotagal UR. Pay for performance alone cannot drive quality. Arch Pediatr Adolesc Med. 2007;161(7):650-655.

13. Grossbart SR. What's the return? Assessing the effect of "pay-for-performance" initiatives on the quality of care delivery. Med Care Res Rev. 2006; 63(1) (suppl):29S-48S.

14. Berthiaume JT, Chung RS, Ryskina KL, Walsh J, Legorrets AP. Aligning financial incentives with "Get With the Guidelines" to improve cardiovascular care. Am J Manag Care. 2004;10(7 pt 2):501-504.

15. Rogliano J. Sampling best practices. Managing delinquent records. J AHIMA. 1997;68(8):28,30.
16. Greene SE, Nash DB. Pay for performance: an overview of the literature. Am J Med Qual. 2009;24;140-163.

17. McMahon LF, Hofer TP, Hayward RA. Physician-level P4P:DOA? Can quality-based payments be resuscitated? Am J Manag Care. 2007;13(5): 233-236.

18. Casalino LP, Elster A, Eisenberg A, et al. Will pay for performance and quality reporting affect health care disparities? Health Aff (Millwood). 2007;26(3):w405-w414.

19. Campbell SM, McDonald R, Lester H. The experience of pay for performance in English family practice: a qualitative study. Ann Fam Med. 2008; 8(3):228-234.

20. Teleki SS, Damberg CL, Pham C., et al. Will financial incentives stimulate quality improvement? Reactions from frontline physicians. Am J Med Qual. 2006;21(6):367-374.

21. Pierce RG, Bozic KJ, Bradford DS. Pay for performance in orthopedic surgery. Clin Orthop Relat Res. 2007;457:87-95.

22. Seidel RL, Baumgarten DA. Pay for performance survey of diagnostic radiology faculty and trainees. J Am Coll Radiol. 2007;4(6):411-415. 